\title{
PARTIAL SUMS OF THE NORMALIZED LOMMEL FUNCTIONS
}

\section{MuRAT ÇAĞLAR AND ERHAN DENIZ}

Abstract. The aim of the present paper determine the ratio of the normalized Lommel functions $\mathscr{L}_{\mu, v}$ of the form (??) to its sequence of partial sums $\left(\mathscr{L}_{\mu, v}\right)_{m}(z)=z+\sum_{n=1}^{m} a_{n} z^{n+1}$ when the coefficients of $\mathscr{L}_{\mu, v}$ satisfy some conditions. Furthermore we investigate the radii of univalency, starlikeness, convexity and close-to-convexity of the partial sums $\left(\mathscr{L}_{\mu, v}\right)_{m}(z)$. Computational and graphical usages of Maple (Version 17) as well as geometrical descriptions of the image domains in several illustrative examples are also presented.

Mathematics subject classification (2010): 30C45, 33C10.

Keywords and phrases: Partial sums, analytic functions, univalent function, Lommel functions, normalized Lommel function, starlike function, convex function, close-to-convex function.

\section{REFERENCES}

[1] A. BARICZ, S. Koumandos, Turán type inequalities for some Lommel functions of the first kind, arXiv:1308.6477.

[2] A. BARICZ, R. SZÁsZ, Close-to-convexity of some special functions and their derivatives, Bull. Malays. Math. Sci. Soc. (in press).

[3] L. Brickman, D. J. Hallenbeck, T. H. MacGregor and D. Wilken, Convex hulls and extreme points of families of starlike and convex mappings, Trans. Amer. Math. Soc. 185 (1973), 413428.

[4] M. ÇAĞLAR, H. ORHAN, On neighborhood and partial sums problem for generalized Sakaguchi type functions, The Scientifc Annals of Al. I. Cuza University of Iasi, (2013), (in press).

[5] M. L. GLASSER, Integral representations for the exceptional univariate Lommel functions, J. Phys. A. 43, 15 (2010), 155207, 4 pp. MR2608275 (2011e:33011).

[6] A. W. Goodman, Univalent Functions, Vol I. Mariner Publ. Comp., Tampa, Florida, 1984.

[7] S. Koumandos, M. Lamprecht, The zeros of certain Lommel functions, Proc. Amer. Math. Soc. 140, 9 (2012), 3091-3100.

[8] L. J. Lin AND S. Owa, On partial sums of the Libera integral operator, J. Math. Anal. Appl. 213, 2 (1997), 444-454.

[9] E. Lommel, Ueber eine mit den Besseischen Functionen verwandte Function, Math. Ann. 9 (1876), 425-444.

[10] Y. MiKi, A note on close-to-convex functions, J. Math. Soc. Japan, 8 (1956), 256-268.

[11] K. Noshiro, On the starshaped mapping by an analytic function, Proc. Imp. Acad. 8, 7 (1932), 275-277.

[12] E. Deniz, H. ORHAN, Some properties of certain subclasses of analytic functions with negative coefficients by using generalized Ruscheweyh derivative operator, Czech. Math. J. 60, 3 (2010), 699713.

[13] E. Deniz, H. ORhan, Certain subclasses of multivalent functions defined by new multiplier transformations, Arab. J. Sci. Eng. 36, 6 (2011), 1091-1112.

[14] H. Orhan and N. YaĞMur, Partial Sums of generalized Bessel functions, J. Math. Inequal. 8, 4 (2014), 863-877.

[15] S. OWA, H. M. SRivastaVA AND N. SAito, Partial sums of certain classes of analytic functions, Int. J. Comput. Math. 81, 10 (2004), 1239-1256. 
[16] V. Ravichandran, Geometric properties of partial sums of univalent functions, arXiv: $1207.4302 \mathrm{v} 1$.

[17] T. Sheil-Small, A note on partial sums of convex schlicht functions, Bull. London Math. Soc. 2 (1970), 165-168.

[18] R. SingH, Radius of convexity of partial sums of a certain power series, J. Austral. Math. Soc. 11 (1970), 407-410.

[19] H. Silverman, Partial sums of starlike and convex functions, J. Math. Anal. Appl. 209 (1997), 221227.

[20] H. Silverman, Partial sums of a class of univalent functions, Tamkang J. Math. 29, 3 (1998), 171174.

[21] E. M. Silvia, On partial sums of convex functions of order $\alpha$, Houston J. Math. 11 (1985), 397-404.

[22] J. Steinig, The sign of Lommel's function, Trans. Amer. Math. Soc. 163 (1972), 123-129.

[23] G. N. Watson, A Treatise on the Theory of Bessel Functions, Second edition, Cambridge University Press, Cambridge, London and New York, 1944. 